\title{
ANÁLISIS EXTERNO PARA EL PROCESO DE IMPLEMENTACIÓN DE LA TELEVISIÓN DIGITAL TERRESTRE INTERACTIVA EN COLOMBIA
}

Julio Omar Ancízar Palacio-Niño1: Universidad Nacional de Colombia. Colombia. jopalacion@unal.edu.co

\section{Ingrid Páez-Parra:}

\section{RESUMEN}

Colombia escogió el estándar europeo (DVB-T) para la aplicación de la televisión digital terrestre (TDT), esto genera un gran progreso no solo en el nivel de transmisión de televisión, sino establece toda una nueva plataforma de servicios y oportunidades. En la actualidad existe una migración hacia el sistema de transmisión y recepción TDT, este esquema de televisión digital, en cualquiera de sus modalidades, terrestre, cable, satelital; ofrece una mejor calidad de imagen y sonido entre otros. De igual manera Suramérica está en proceso de migración hacia un sistema de transmisión digital de televisión, en su mayoría bajo el estándar ISDB-Tb lo que puede generar un interesante marco de desarrollo para el país y la situación actual frente a sus vecinos. Con el fin de generar estrategias y recomendaciones para la implementación de contenidos educativos en Colombia por medio de la televisión digital terrestre, se analizará el entorno externo a Colombia el cual puede afectar de manera positiva o negativa el proceso. Este análisis sintético permitirá encontrar las oportunidades que tiene Colombia para el proceso de implementación como también encontrar posibles amenazas frente a otros procesos similares implementados en la región que pueden afectar su desarrollo.

PALABRAS CLAVE: DVB - Educación - Interactividad - Televisión Digital

\footnotetext{
${ }^{1}$ Autor correspondiente

Julio Omar Ancízar Palacio-Niño: Ingeniero de Sistemas, Candidato a Magister en Ingeniería de Telecomunicaciones. Universidad Nacional de Colombia. Bogotá D.C. (Colombia).

Correo: jopalacion@unal.edu.co
} 


\title{
EXTERNAL REVIEW PROCESS FOR THE IMPLEMENTATION OF INTERACTIVE DIGITAL TERRESTRIAL TELEVISION IN COLOMBIA
}

\begin{abstract}
Colombia has chosen the European standard (DVB-T) for the implementation of digital terrestrial television (DTT), this generates a lot of progress not only in television transmission standard, but provides a whole new platform of services and opportunities. At present there is a migration to the transmission and reception system TDT, this scheme of digital television in all its forms, terrestrial, cable, satellite, offering a better quality picture and sound among others. Similarly, South America is in the process of migration to a digital TV transmission, mostly under the standard ISDB-Tb that can generate an interesting development framework for the country and the current situation against its neighbors. In order to generate strategies and recommendations for the implementation of educational content in Colombia through digital terrestrial television will analyze the external environment to Colombia which can affect the process positively or negatively. This summary analysis will find the opportunities to Colombia for the implementation process as well as identify potential threats to other similar processes implemented in the region that can affect their development.
\end{abstract}

KEY WORDS: DVB - Education - Interactivity - Digital Television

\section{INTRODUCCIÓN}

La televisión, tienen una amplia penetración en nuestra sociedad colombiana, como quedo demostrado en la encuesta realizada por la empresa consultora IPSOS NAPOLEÓN FRANCO a petición de la Comisión Nacional de Televisión para el estudio de la televisión digital terrestre en agosto de 2008, arrojando resultados como estos: (IPSOS - NAPOLEÓN FRANCO, 2008)

a) El 99\% de los hogares colombianos tienen televisor

b) 7 de cada 10 colombianos mayores de 12 años ven televisión a diario

c) 6 de cada 10 personas consideran la televisión importante en su vida diaria

d) Para los estratos bajos es de mayor importancia la televisión, como medio de entretenimiento, educación e informativo.

e) El $60 \%$ de los hogares colombianos piensan migrar rápidamente a la televisión digital cuando se empiece a implementar. 
La Comisión Nacional de Televisión ${ }^{2}$ define como Televisión Digital:...la tecnología que utiliza para transmitir su señal. Contrario a la televisión tradicional, que envía sus ondas de manera analógica, la digital codifica sus señales de forma binaria..."3 .

A partir de esta premisa Colombia selecciono el estándar europeo (DVB-T) para la aplicación de la televisión digital terrestre (TDT) frente a los otros estándares evaluados como el estándar japonés - brasilero (ISDB-Tb), americano (ATSC) o el estándar chino (DMB-T), estableciendo toda una nueva plataforma de servicios y oportunidades que estará al alcance de todos los colombianos. (Comisión Nacional de Televisión (CNTV), 2009)

Al utilizar un sistema de transmisión digital se obtiene grandes beneficios con respecto al sistema análogo, entre estos podremos indicar una calidad de imagen superior similar a la de un video en formato DVD, toda las transmisiones son en formato 16:9, recepción de audio de alta calidad tipo Dolby AC-3, en formato 5.1 y opciones de multilenguaje, capacidades de Multiplexación con lo cual permite aprovechar de una mejor manera el espectro radioeléctrico, posibilidad de recepción en dispositivos móviles en movimiento con una excelente calidad de imagen y sonido, utilización de una menor cantidad de energía eléctrica tanto en los transmisores como en los receptores, entre otras ventajas. (Digital Video Broadcasting (DVB), 2009; Grupo de Trabajo 4 del Foro Técnico de la televisión digital, 2005)

Al tener estas ventajas tecnológicas cambia la categorización de televidente pasivo a la de un televidente activo, capaz de interactuar con los programas que está viendo en televisión, todo gracias a la incorporación de la interactividad; esta interactividad abre nuevos campos de desarrollo y uno de estos es el t-learning, ofreciendo una interesante herramienta complementaria al proceso educativo, dando la posibilidad al usuario de interactuar con los programas, acceso a material complementario, profundizar en la temática vista y opinar sobre lo que observa.

\section{DESARROLLO}

\subsection{Televisión digital y región}

El panorama regional de Colombia con respecto a la adopción del estándar de televisión digital es adverso, considerando que es el único país de Suramérica en adoptar el estándar DVB-T, después de que Uruguay cambiara del estándar DVB-T al ISDB-Tb, lo cual le puede generar inconvenientes regionales pero también oportunidades internas.

Para determinar en qué situación se encuentra Colombia y ver cómo puede tomar

\footnotetext{
2 Organismo de derecho público dedicado a desarrollar y ejecutar los planes y programas del Estado en el servicio público de televisión (Colombia).

${ }^{3}$ Comisión Nacional de Televisión (CNTV), "Televisión Digital Terrestre - Informe," Comisión Nacional de Televisión (CNTV), 2008
} 
experiencias de migración de otros países que puedan servir como referentes, se analizaron dos países que en la actualidad adoptaron el estándar DVB-T (España, Italia) y dos países regionales que adoptaron el estándar ISDB-Tb (Brasil, Argentina) y considerar los caminos que realizaron estos países para complementar el proceso desarrollado en Colombia, precisar que amenazas externas se podrían presentar y como Colombia podría contrarrestar esta situación particular.

\subsection{España}

España inicio su proceso de implementación de la televisión digital bajo el estándar DVB-T en el año 1999 por medio de una licencia otorgada por concesión a la empresa Onda Digital S.A.e implementando un proceso de promoción y emisión bajo la modalidad de pago en la marca Quiero TV. Ya para el año 2002 empezaron las emisiones de dos canales abiertos como lo son Veo TV y Net TV.

Debido a la poca variedad en la programación y la fuerte competencia que representa la televisión por satélite y por cable, los cuales ofrecían una mayor variedad de programación, el proyecto fracaso declarándose la empresa en bancarrota en el año 2002.(Urquiza García, 2007)

Para el año 2005 se hizo un proceso de re-lanzamiento de la televisión digital por parte del estado y siendo la RTVE el principal organismo impulsor de la televisión digital, cambiando la estrategia de servicio pago por un servicio gratuito, fortaleciendo las siguientes características:
a) Emisión de contenidos novedosos
b) Capacidad multi-idioma
c) Servicios interactivos

\subsection{Italia}

En 1998 Italia inicio sus intenciones de implementar el sistema digital de televisión bajo el estándar DVB-T, pero a diferencia de España, Italia desarrolla un sistema de implementación hibrido entre el estado y el sector privado creando un organismo multilateral llamado DGTVi, encargado del desarrollo de la televisión digital terrestre.

En 2004 empieza el proceso fuerte de implementación del estándar en todo el territorio nacional por parte del estado representado en la RAI y de la mano con su contraparte privada Mediaset, apostando a dos mercados claramente diferenciados: televisión abierta y PPV (pay per view), siempre teniendo en cuenta la implementación de contenidos interactivos para ambas plataformas.

A diferencia de otros países, Italia tiene una infraestructura pobre para la televisión 
una penetración del 15\%, el resto solo recibe televisión abierta (terrestre). (DGTVi, 2006)

Un componente diferenciador de otros países de la unión europea fue la apuesta al desarrollo de la interactividad bajo la plataforma MHP, que permitiera una mayor integración, acceso universal a la información, entre otros beneficios; como política de masificación el estado italiano destino recursos (subsidios) para la adquisición de decodificadores con capacidades interactivas. (Morello, 2007)

A nivel público se ofrecen varios servicios interactivos como el servicio de lanzadera, RaiNews 24, t-goverment, entre otros y de manera privada se ofrece el servicio de PPV por medio de la adquisición de tarjetas prepago. (DGTVi, 2006).

\subsection{Argentina}

En 1998 Argentina adopto el estándar ATSC para la televisión digital, pero este estándar fue utilizado muy poco, por lo que en el 2006 se decidió revocar la decisión y adoptar como nuevo estándar de televisión digital el ISDB-Tb o más conocido como estándar japonés-brasilero.

La implementación del sistema de televisión digital, en argentina conocido como TDA (televisión digital abierta) es un proyecto netamente estatal que busca motivar la participación, ofrecer servicios interactivos, entre otros. (Televisión Digital Abierta (TDA), 2011). Al ser un sistema netamente estatal se busca que el sistema ofrezca gratuidad, cobertura nacional, TV móvil, mayor participación en la televisión, desarrollo de la industria nacional.

Dado a que argentina es un país extenso y existen regiones con muy baja densidad poblacional, la televisión digital será afrontada desde dos segmentos, la TDA que será por el sistema terrestre de televisión digital bajo el estándar ISDB-Tb y el sistema de televisión digital satelital (TDS) el cual será de acceso gratuito en el cual se está realizando en el satélite AMC-6 bajo el estándar DVB-S2.

Para motivar el procedo de implementación se desarrollo un plan operativo llamado "mi TV digital" el cual le otorga un kid de recepción de la señal digital a sectores de la sociedad puedan sufrir de exclusión en el proceso de migración. (Mi TV Digital, 2011)

En la parte de interactividad usa la plataforma GINGA, la cual se encuentra en un proceso de estudio por parte de la Universidad de la Plata para ser implementada.

\subsection{Brasil}


Desde el año 1999, Brasil por medio de un comité estudió la implementación de un sistema de televisión digital para el territorio nacional, dando como resultado en el año 2003 el SBTVD (Sistema brasilero de televisión digital) el cual fuera un eje de investigación y desarrollo nacional.

El estándar adoptado por Brasil es una variación técnica del estándar ISDB-T sugerida por el comité investigador, a esta variación del estándar se le conoce como SBTVD-T o ISDB-Tb; el sapecto fundamental en la diferenciación entre el estándar original y la variación brasilera es la adopción de una compresión bajo el estándar MPEG-4 y la plataforma interactiva llamada GINGA, como una plataforma libre. (Anatel, 2011)

Ya en el año 2006 se da de manera oficial la implementación del estándar de televisión digital enfocado a una televisión libre y gratuita, teniendo en cuenta la alta definición, movilidad, interactividad, flexibilidad.

A pesar de que el estándar ISDB-T solo era implementado por tres países y no existía una economía a escala para la producción y comercialización de equipos capaces de recibir la señal televisiva, Brasil le apuesta a una investigación y desarrollo de los equipos, lo que al principio fue bastante duro, donde para el año 2008 los STB mas económicos estaban alrededor de los US\$ 300.

La poca implementación en otros países fue una situación definitiva para que Brasil asumiera el liderato del estándar y lo convirtiera en una gran fuente de investigación y desarrollo tecnológico ofreciéndolo como una alternativa y eje de desarrollo regional, promocionándolo en toda la región (Suramérica). (Site Oficial da TV Digital Brasileira, 2011).

\subsection{Análisis Sintético}

Para determinar las oportunidades externas que cuenta Colombia en este proceso de implementación se dio a responder las siguientes preguntas basadas en un análisis DOFA:

a) ¿Qué obstáculos impedirían el buen desempeño y desarrollo de la implementación de contenidos educativos en el sistema de TDT interactivo en Colombia? (amenazas)

b) ¿Qué eventos o tendencias del entorno (tecnológico, económico, educacional), puede aprovecharse como beneficio para la implementación de contenidos educativos en el sistema de TDT en Colombia? (oportunidades).

\subsection{Amenazas}


televisión digital en Suramérica no es el más propicio para el desarrollo regional, ya que toda Suramérica adopto el estándar ISDB-Tb (brasilero - japonés), exceptuando Colombia que adopto el estándar DVB-T; Guyana y Surinam no han definido aun estándar. Está situación deja aislada a Colombia en la posibilidad de intercambio de tecnología, innovación, contenidos, entre otros.

Crecimiento es otras modalidades de masificación: en la actualidad el crecimiento de otras formas de masificación del servicio de televisión como lo son el Cable, Satelital y el IPTV tienen cada vez más penetración entre la población, principalmente en los centros urbanos, donde el índice va desde un $70 \%$ hasta un $90 \%$. Esta masificación es causada por la disminución en los precios del servicio y la gran oferta de programación y particularmente en la penetración en el territorio nacional de este servicio por medio de operadores satelitales. También cada vez disminuyen los precios de servicios digitales de televisión con lo denominado (televisión avanzada), tanto en los cable operadores como ya está por defecto en los servicios satelitales y de IPTV.

Inconsistencias para el uso de otras tecnologías, (IPTV, móvil, cable, satelital): la principal debilidad es el proceso llevado a cabo en estos momentos, el cual está centrando todos sus esfuerzos realizar transmisiones en el sistema DVB-T, dejando un vacio del proceso a seguir para los usuarios pertenecientes a sistemas de televisión por suscripción. Aunque el estándar DVB tiene especificaciones para cada uno de estos sistemas de transmisión como se vio anteriormente, en los procesos realizados por la CNTV no se especifica el camino a seguir para las compañías prestadoras de este servicio y mucho menos a los usuarios, que son el $70 \%$ de la población total que tiene acceso a la televisión en Colombia.

Inconsistencias para el uso de otras tecnologías para el canal de retorno: al igual que en el caso de los otros sistemas de transmisión, no hay claridad en el medio de retorno que podría ser utilizado por estos servicios interactivos, aunque el estándar DVB cuenta con varias especificaciones sobre interactividad y canal de retorno, no hay en estos momentos claridad para su implementación dejando como primera opción el acceso al internet, por lo que garantizar que el usuario final pueda establecer una interacción con el canal que ofrece el contenido, obliga al usuario tener acceso a una de estos canales de retorno, lo que puede desmotivar su uso.

\subsection{Oportunidades}

Mercado a escala de equipos receptores: una de los puntos a favor para la selección del estándar europeo es el mercado a escala que ofrece para el usuario final en decodificadores y IDTV, lo que puede generar un acceso económico a los equipos.

Tecnología ya implementada en otros países: el estándar DVB-T al ser el más difundido a nivel mundial, con 35 países implementando el estándar ofrece varios marcos de referencia en le proceso de migración que se tiene que realizar así como 
una validación de equipos y procedimientos, con una gran oportunidad en el intercambio de experiencias en el proceso de migración y procesos de transferencia de conocimiento.

Oportunidad de apoyo de entidades externas: el estándar tiene la posibilidad de apoyo logístico y procedimental del proceso de implementación, como la organización DVB, Unión Europea, España a través del ministerio de industria, turismo y comercio.

Nuevos mercados para los proveedores de servicios: en la actualidad Colombia es reconocida como una potencia regional en la producción de contenidos televisivos, y al ser el estándar DVB-T el mas difundido a nivel mundial, existe la posibilidad de exportar contenidos televisivos e inclusive interactivos a países fuera de la región.

Introducción del nuevo estándar DVB-T2: el estándar DVB-T2 es la nueva versión del estándar DVB-T lo que permitiría en un futuro migrar fácilmente a este nuevo estándar el cual ofrece mayores anchos de banda y mayores posibilidades de servicios, en la actualidad se encuentra en periodo de pruebas en varios países como el Reino Unido, Suecia, Italia, Rusia, etc.

\section{CONCLUSIONES}

Después del análisis de la situación externa que presenta Colombia frente a la implementación del sistema de televisión digital se pueden considerar las siguientes conclusiones:

a) El estándar DVB-T ofrece a Colombia una gran oportunidad de intercambio tecnológico, comercial, etc con los países donde este estándar ya esta implementado, así como un gran aprendizaje de las experiencias de ellos, además se desarrolla el sector de contenidos en el cual es país ha venido creciendo ampliamente.

b) Está tecnología al ser la mas difundida a nivel mundial tiene un gran mercado a escala ya consolidado por lo que los equipos necesarios para su migración, en especial los STB y los IDTV estarán a precios bastante asequibles a todo público, logrando una masificación rápida.

c) Colombia deberá buscar los mecanismos que le permitan establecer convenios de intercambio de contenidos con la región, y así no quedar aislado, ya que es el único país que no adopto el estándar ISDB-Tb. 
conlleva la adopción del estándar para involucrarse en el proceso investigativo y lograr generar conocimiento adaptado a las necesidades del país, y lograr apropiarse de la tecnología y no ser un simple consumidor.

\section{REFERENCIAS}

ANATEL. (2011). TV Digital alcança 46\% da população brasileira. Recuperado el 10 de Agosto de 2011, de

http:// www.anatel.gov.br/Portal/exibirPortalPaginaEspecialPesquisa.do?acao=\&ti poConteudoHtml=1\&codNoticia=22469.

CNTV. (2009). Así se eligió el estándar de televisión digital terrestre para Colombia. Recuperado el 10 de Agosto de 2011, de http://www.cntv.org.co/cntv_bop/tdt/contenido16.html.

DGTVi. (2006). The strategy of commercial broadcasters in de digital transition. En Digital Terrestrial TV in Italy. Recuperado el 10 agosto de 2011, de http://www.dtt.ba/prezentacije/prezentacije_4/Viggiani_The\%20transition\%20to\% 20DTT\%20in \%20France.pdf.

GRUPO DE TRABAJO 4 DEL FORO TÉCNICO DE LA TELEVISIÓN DIGITAL. (2005). Televisión Digital: Adaptarse hoy para la nueva televisión. Trabajo presentado al Foro Técnico de la televisión digital. Madrid.

Ipsos - Napoleón, F. (2008). Estudio de Usos y Hábitos - informe metodológico. Bogotá: Comisión Nacional de Televisión (CNTV).

MI TV DIGITAL (2011). ¿Cómo acceder a la TDA? Recuperado el 10 de Agosto de 2011, de http://www.mitvdigital.gob.ar/mitvdigital/elplan?t=El\%20Plan\&tag=mitvdigital_elprograma\&order=fecha_asc\&opt=1.

Morello, A. (2007). Digital Television in Italy. Radiotelevisione Italiana (RAI). Recuperado el 10 de Agosto de 2011, de http:// forbrugerelektronik.dk/fileadmin/user_upload/forbrugerelektronik/BFE/k onferencer/2-Alberto-Morello-RAI.ppt.

Site oficial da tv digital brasileira (2011). TV Digital: Programas buscam capacitar programação e difundir conteúdos interativos. Recuperado el 10 de agosto de 2011, de http://www.dtv.org.br/index.php/tv-digital-programas-buscam-capacitarprogramacao-e-difundir-conteudos-interativos/.

TDA. (2011).¿Qué es la TDT? Recu pera d o el 10 de agosto 2011, de http://www.tvdigitalargentina.gob.ar/tvdigital/tvdigital?t=\&tag=tv_digital\&order =fecha_asc\&opt $=2$. 
Urquiza García, R. (2007). Televisión Digital Terrestre: modelos de negocio en el mercado europeo. Global Media Journal, 4.

\section{Julio Omar Ancízar Palacio-Niño}

Ingeniero de Sistemas de la Universidad Nacional de Colombia. Candidato al título de Magister en Ingeniería de Telecomunicaciones en la Universidad Nacional de Colombia. Participo como ponente en el simposio "Las TIC y el Cambio Climático" Ponente en el Encuentro Nacional de Investigación y Desarrollo (ENID), (2010) Bogotá D.C. (Colombia). Ponente en el II Congreso Internacional de Gestión Tecnológica e Innovación (2010). Ha sido Docente Auxiliar en la Universidad Nacional de Colombia en las áreas de Programación de Computadores y Métodos Numéricos (2010) y actualmente soy Docente Cátedra de las asignaturas de Investigación de Operaciones y Modelos de Toma de Decisiones en la Institución Universitaria Politécnico Gran colombiano. 